\title{
Computational complexity reduction in HEVC intra prediction using dual tree complex wavelet transform and holoentropy
}

\author{
Swati Vinod Sakhare ${ }^{1}$, Dr. Mrs. Upena D. Dalal ${ }^{2}$
}

${ }^{1}$ Research Scholar, Department of Electronics Engineering, Sardar Vallabhbhai National Institute of Technology, Surat, Gujarat, India ${ }^{2}$ Professor, Department of Electronics Engineering, Sardar Vallabhbhai National Institute of Technology, Surat,Gujarat, India.

${ }^{1}$ swatisakhare123@rediffmail.com ${ }^{2}$ udd@eced.svnit.ac.in

\begin{abstract}
High efficiency video coding (HEVC) is the newest video codec to increases the coding efficiency of its ancestor H.264/Advance Video Coding at the cost of highly increased computational complexity. In this paper, a novel method using Dual tree Complex Wavelet Transform is proposed to reduce the computational time consumption in HEVC Encoding. DTCWT provides better directional selectivity. By using DTCWT, total intra prediction modes are reduced from 35 modes to 7 modes including DC and Planer mode. The encoding process in HEVC system is performed using clustered entropy computing, which distinguishes the video information has useful outliers. The pixel variations under varying frames are clustered based on the interestingness and the outliers are removed using an advanced entropy principle called as holoentropy. Compared to the current state of the art algorithms, the experimental results show that this scheme is computationally simple and achieves superior reconstructed video quality at less computational complexity.
\end{abstract}

Keywords- Fast Encoding, DTCWT, High Efficiency Video Coding, Quadtree-Based Coding Unit Partitioning, Cluster Holoentropy, CABAC.

\section{Introduction}

Today's world is called as "digital or electronic age", where mobile phones not only make phone calls but are also used for web browsing, sending emails, watching/capturing videos, transfer data, navigation purposes and take pictures. Data is stored on rewritable DVDs, Blu-ray discs and hard disks which are light weight, portable with huge space for storage. Furthermore, the current trend in most portable devices with embedded digital cameras is to include the capability of encoding and decoding high-resolution digital video streams.

Despite the recent evolution in portable devices, particularly in terms of communications technology and computational power, the limited battery capacity still imposes major constraints in multimedia applications demanding high computational power, such as those dealing with video encoding. In such cases, the user experience might be limited by the reduced battery capacity. Furthermore, even in those cases in which battery capacity is not an issue, encoding and decoding high-resolution digital video streams in real time is still a challenge, especially when considering the computational requirements of the most recent video coding standards [2]. Hence, research is still going on in the field of compression techniques to enable real-time data transmission using fewer resources.

Furthermore, video buyers progressively request top quality (HD) and (UHD) recordings to encounter better visual quality. The information amount for HD/UHD video is tremendous because of the higher video goals and edge rate.

The demands for video with higher quality and resolution are however always increasing. As an example, HDTV is well on its way to replace standard definition TV [4]. This demand implies a need for new standards with higher coding efficiency. In addition, video content is to a greater extent than before being streamed over the Internet, to a variety of platforms. Since bandwidth is a limited resource, this gives another incentive for developing more efficient standards. As a consequence, two of the major video coding standardization organizations, the ITU-T Video Coding Experts Group (VCEG) and the ISO/IEC Moving Picture Experts Group (MPEG), established a Joint Collaborative Team on video coding (JCT-VC) in January 2010, and issued a Call for Proposals (CfP). The new project was given the name "High Efficiency Video Coding" (HEVC) [4]. The HEVC standard is intended to provide significantly better compression capability than the existing H.264/AVC standard. HEVC achieves $40 \%-50 \%$ bit rate reduction in comparison with its predecessor, the H.264/AVC video coding standard, at the same subjective image quality. However, to reach such goal, HEVC incorporates several new tools, which increases the encoder computational complexity in comparison to H.264/AVC High Profile [1]. 
ITU-T and ISO/IEC are the principle institutionalization bodies which have institutionalized all HEVC's predecessor guidelines in numerous years. They have used a $16 \times 16$ macroblock as an essential handling unit in HEVC's precursor. Each edge is part into macroblocks. Each macroblock includes one $16 \times 16$ square of luma parts to speak to the splendor and two $8 \times 8$ squares of chroma segments to allude the shading in the 4:2:0 chrominance subsampling groups. In any case, ordinary HD and UHD recordings have numerous bigger edge areas than the macroblock, and those districts can speak to the equivalent moving data. The macroblock is utilized as an essential preparing unit for regular HD and UHD recordings, lots of bits are important to flag the expectation data. Correspondingly, the change square size is bigger than the macroblock size. Consequently, HEVC uses a bigger square size as a fundamental handling unit called CTU for intraoutline or between outline expectation and change coding. HEVC can deftly parcel the video outline into a few square CTUs of $2 \mathrm{~L} \times 2 \mathrm{~L}$ tests, where $\mathrm{L} \in\{4,5,6\}$. The encoder deftly picks a reasonable estimation of $\mathrm{L}$ for proposed application to have the best ex- change off between coding execution and cost.

\section{A. High Efficiency Video Coding}

HEVC has the same basic structure as previous standards such as H.262, H.263, MPEG-4 Visual and H.264/AVC but contains some improvements such as:

- More flexible partitioning (from large to small partition sizes)

- Greater flexibility in prediction modes and transform block sizes

- More sophisticated interpolation and deblocking filters

- More sophisticated prediction and signaling of modes and motion vectors

- Features to support efficient parallel processing.

The result is a video coding standard that can enable better compression, at the cost of potentially increased processing power. The main goal of HEVC is to double the compression efficiency of his predecessor, the H.264/AVC. This means:

- At the same picture size and quality, an HEVC video sequence should occupy less storage, or transmission capacity, than the equivalent H.264/AVC video sequence.

- Resolution of an HEVC video sequence should be higher than the corresponding H.264/AVC video sequence.
Several studies have concluded that HEVC can deliver similar quality to H.264 at approximately half the bit rate.

HEVC is based on the same general structure as previous standards. A source video which consists of a sequence of video frames is encoded (or compressed) by the HEVC Video Encoder (Fig.1), creating a compressed video bitstream. After that, the compressed bitstream is either stored or transmitted. After the transmission, the Video Decoder decompresses the bitstream, creating a sequence of decoded frames.

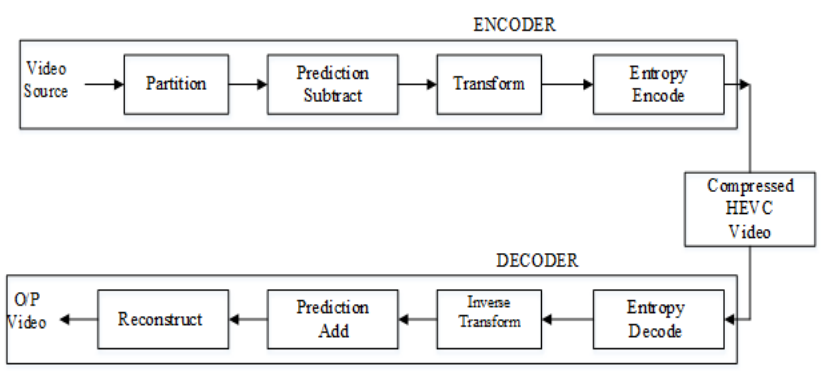

Fig.1. HEVC Structure

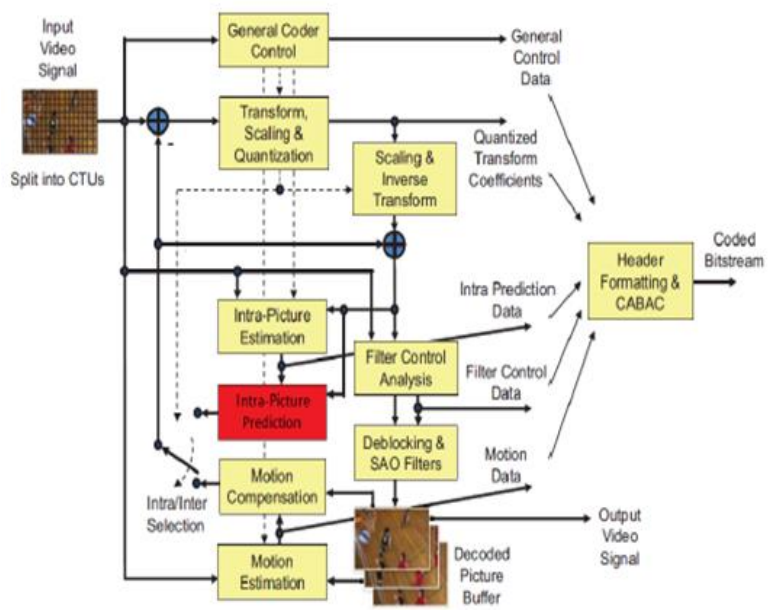

Fig.2. HEVC Encoder Structure [6]

Fig. 2 shows the HEVC Decoder. The steps carried out by a Video Encoder include:

- $\quad$ Partitioning each picture into multiple units

- $\quad$ Predicting each unit using inter or intra prediction, and subtracting the prediction from the unit

- Transforming and quantizing the residual (the difference between the original picture unit and the prediction)

- $\quad$ Entropy encoding the output, prediction information, mode information and headers.

The HEVC Video Decoder is shown in Fig.3. It reverses the steps:

- $\quad$ Entropy decoding and extracting the elements of the coded sequence

- $\quad$ Rescaling and inverting the transform stage 
the output of the inverse

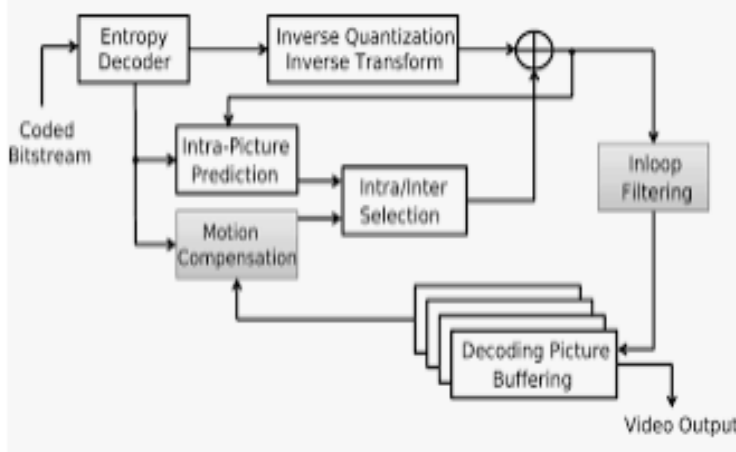

Fig.3. HEVC Decoder Structure [33]

HEVC supports highly flexible partitioning of video sequences. Each coded video frame, or picture, is partitioned into Tiles and/or slices, which are further partitioned into Coding Tree Units (CTUs). The CTU is the basic unit of coding, and can be up to $64 \times 64$ pixels size. A CTU can be subdivided into square regions known as Coding Units (CUs) using a quadtree structure. CU can have various sizes but they have to be square shaped.

A Coding Unit (CU) is partitioned into one or more Prediction Units (PUs). PU is the basic unit for prediction, where the largest allowed PU size is equal to the CU size. Other allowed PU sizes depend on prediction type, where asymmetric splitting options for inter-prediction is also considered. HEVC partition can be seen in Fig.4. Then, each PU is predicted using Intra or Inter prediction.

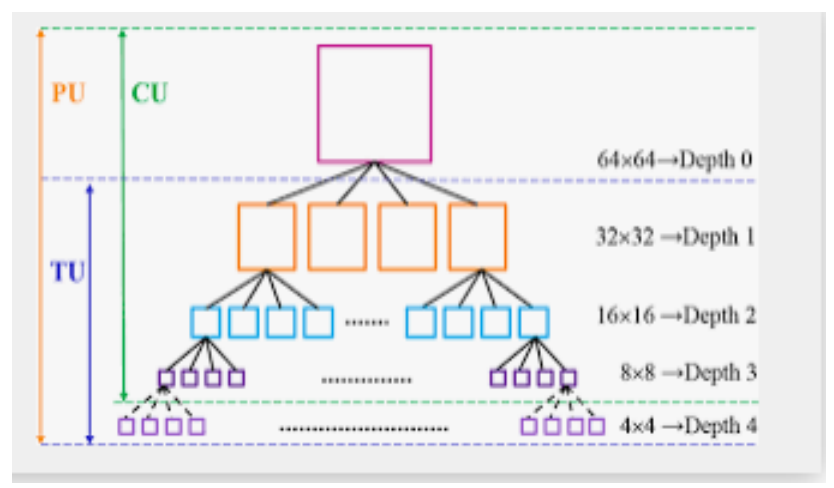

Fig.4. HEVC Partition [32]

So, the general outline of the coding structure is formed by various sizes of CUs, PUs and TUs in a recursive manner, once the size of the Largest Coding Unit (LCU) and the hierarchical depth of CU are defined. CU can be expressed as a recursive quadtree representation, where the leaf nodes of CUs can be further split into PUs or TUs.

Thus, CU size can be $64 \times 64,32 \times 32,16 \times 16$, and $8 \times 8$ at profundity 0 , profundity 1 , profundity 2 , and profundity

3,individually. To characterize CU size or profundity, HEVC begins a preliminary encoding which incorporates two primary capacities called the RD cost estimation and correlation in top-down and base up way, individually. In the top-down RD cost computation of a $64 \times 64 \mathrm{CTU}$, the RD costs for all conceivable $85 \mathrm{CUs}$ are determined in a preorder traversal of the quad tree, if the greatest $\mathrm{CU}$ profundity is 3 .

In subtleties, there are $1,4,16$, and 64 CUs at profundity 0 , profundity 1 , profundity 2 , and profundity 3 , separately, and the complete number of CUs is P3 i=0, $4 \mathrm{I}=$ 85 CUs. Subsequent to computing the RD costs for four kids CUs of each parent CU, HEVC goes to the RD cost correlation which choose whether a parent $\mathrm{CU}$ is part or not by looking at the RD cost of parting and non-parting states of parent CU. At that point, HEVC changes to the $\mathrm{RD}$ cost estimation or performs correlation once more, contingent upon the situation of parent CU. In this way, there are 85 computations and 21 examinations in the topdown RD cost estimation and base up RD cost correlation of a $64 \times 64 \mathrm{CTU}$, individually. After at long last looking at a root CU at profundity 0 with its four kids CUs at profundity 1 , the best CU quad tree structure of a CTU with the least RD cost is picked among 83,522 potential quad tree structures.

\section{B. Terminology for Computational Complexity}

Computational complexity is a term used to describe the amount of calculations performed in a task. The computational complexity refers to the calculations performed in the whole encoding process or in a specific part of it. As the number of computations affects directly the total time of processor usage, computational complexity is always measured in terms of processing time.

Computational complexity reduction is a term used to describe those methods that yield fixed decreases in the computational complexity of a task. Once applied to a determined algorithm, a computational complexity reduction technique is able to decrease the amount of computational resources required to complete the encoding process to a level that is dependent upon the video source characteristics.

\section{C. Importance of computational complexity reduction in HEVC/H.265}

HEVC/H.265 has very efficient compression methods, which allow it to compress video much more efficiently than older standards and provide more flexibility for application to a wide variety of network environments. To achieve highly efficient compression, the computational cost associated with it is also very high. This is the reason why, these increased compression efficiencies cannot be exploited 
across all application domains. Resource constrained devices such as cell phones and other embedded systems use simple encoders or simpler profiles of the codec to tradeoff compression efficiency and quality for reduced complexity [5]. Video coding standards specify the decoding process and bit stream syntax of the compressed video. The encoding process or the process of producing a standard compliant video is not specified. This approach leaves room for innovation in the encoding algorithm development.

\section{D. Intra Prediction in HEVC}

Intra picture prediction (Fig. 5) operates according to the TB size, and previously decoded boundary samples from spatially neighboring.

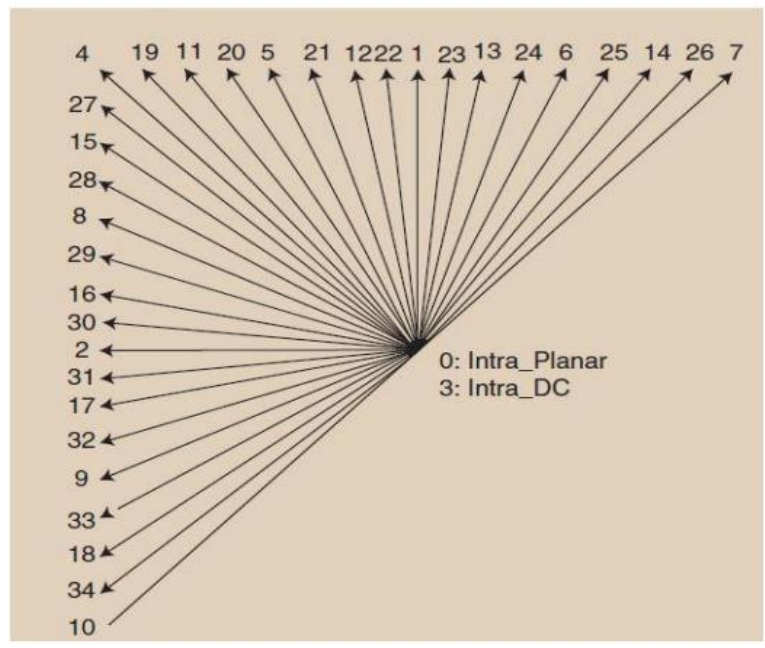

Fig 5: Luma intra prediction modes of HEVC

TBs are used to form the prediction signal. Directional prediction with 33 different directional orientations is defined for TB sizes from $4 \times 4$ to $32 \times 32$.

Alternatively, planar prediction and DC can also be used. For chroma, the horizontal, vertical, planar, and DC prediction modes can be explicitly signaled, or the chroma prediction mode can be indicated to be the same as the luma prediction mode. One of several coding types, depending on the slice type, can code each CB. Similar to H.264/MPEG-4 AVC, intra picture predictive coding is supported in all slice types. HEVC supports various intra picture predictive coding methods referred to as Intra-Angular, Intra-Planar, and Intra-DC. Figure1 shows the luma intra prediction modes of HEVC [28].

HEVC intra prediction employs up to 35 modes for each intra coding block, 33 angular modes which predict the blocks texture at specific directions, plus DC and planar modes for none or less textured areas, based on the average of reference pixels. This time increase in number of modes, which results in a more accurate prediction of the texture, but at the same time demanding more processing power. As applying rate distortion optimization (RDO) to 35 candidates to select the most suitable one would be a very time and energy consuming process as shown in TABLE I .

TABLE I. Current Problem-Complexity for HEVC [30]

\begin{tabular}{|c|c|c|c|}
\hline $\begin{array}{c}\text { Size of } \\
\text { PB }\end{array}$ & $\begin{array}{c}\text { No. of PBs in a } 64 \times \\
\text { 64 CU }\end{array}$ & $\begin{array}{c}\text { No. of Modes to be } \\
\text { tested in each PB }\end{array}$ & $\begin{array}{c}\text { Total No. of Modes } \\
\text { to be tested at this } \\
\text { level }\end{array}$ \\
\hline $32 \times 32$ & 4 & 35 & 140 \\
\hline $16 \times 16$ & 16 & 35 & 560 \\
\hline $8 \times 8$ & 64 & 35 & 2240 \\
\hline $4 \times 4$ & 256 & 35 & 8960 \\
\hline & & Total & 11900 \\
\hline
\end{tabular}

HEVC suggests a two-step approach for intra mode decision. First, a rough mode decision (RMD) process examines all the 35 modes to narrow down the final candidate modes for RDO. Then the RDO process selects the best mode among the limited RMD candidates. So, it is very clear that though $\mathrm{HEVC}$ is efficient as compared to H.264 but at the same time with more computational complexity.

This Time complexity problem in HEVC as shown in Tabel1 motivates to propose efficient algorithm using Dual tree Complex Wavelet Transform along with Holoentropy to reduce time complexity of the HEVC Encoder while maintaining good decoded video quality output.

\section{Related Work}

This section presents an overview of the state-of-the-art research on computational complexity of HEVC video coding process. Even though the HEVC standard have been recently launched, there are already some works published in the literature presenting methods to reduce the encoder's computational complexity. Most of these works aims at decreasing the computational complexity involved in the definition of the new frame partitioning structures, especially the Coding Trees, PUs and RQTs, and apply different techniques to determine the best configuration without testing all possibilities using an RDO process.

HEVC conveys a twofold coding effectiveness when contrasted with its first coding standard to be specific, H.264/Advance Video Coding (AVC) [2]. In particular, HEVC accomplishes the bit rate sparing of almost half under the equivalent visual quality contrasted with the H.264/AVC. Thus, HEVC turns into a famous video codec. HEVC brings such a critical coding proficiency on account of its development coding highlights, for example, various 
levelled quadtree-based parcelling, 33 directional modes for intra-picture forecast and streamlined in-circle deblocking separating, and so forth. Then again, these coding highlights are profoundly costly as far as computational multifaceted nature and equipment necessities. Because of this, HEVC battles to acknowledge HD/UHD video conveyance progressively applications, since most recent couple of years, existing investigations have exhibited numerous arrangements on alleviating the computational weight of HEVC. A large portion of these arrangements underscore a progressive quad tree-based coding unit (CU) apportioning due to the heaviest heap of recursive $\mathrm{CU}$ dividing in the HEVC encoder. In a progressively thorough CU dividing of HEVC, the encoder right off the bat begins a preliminary encoding which incorporates two procedures: top-down rate bending (RD) cost count and base up RD cost correlation with locate an ideal quad tree parcelling design dependent on RD execution gain for each coding tree unit (CTU). To get an ideal CU apportioning design, each conceivable mix of CU size, forecast unit (PU) modes, and change unit (TU) sizes are thoroughly inspected. Because of this, the preliminary encoding, particularly the RD cost figuring, is the most time-consuming module of HEVC, over $80 \%$ in the HEVC test model (HM) [3]. To handle this issue, the vast majority of the examinations intend to accomplish the quick calculation by supplanting a comprehensive CU parceling with a straightforward activity under the immaterial RD misfortune. To effectively spare the computational time of HEVC, there are two primary sorts of research concentrating on intricacy decrease in HEVC intra-coding and inter coding. In light of the new highlights of HEVC intra-coding, for example, two dividing modes in quad treebased parceling and 35 intra expectation modes, the past answers for intra-coding [7] center around intra-mode choice and CTU size forecast. For inter-coding, a few tedious modules, for example, quad tree-based $\mathrm{CU}$ parcelling [8]-[20], CU mode estimation [21], and movement estimation (ME) [22] have been improved to accomplish a low unpredictability encoder. In explicit, there are two gatherings on the quick between coding calculations for quad tree-based $\mathrm{CU}$ parcelling. One is a measurable based quick calculation, and the other is a learning-based quick calculation. The early factual based methodologies have measurably chosen $\mathrm{CU}$ size by watching the idea of unique square parceling in HEVC, for example, in [8]-[12]. These methodologies decide some CU-related highlights and spatiotemporal-based hard limit to choose CU size without circling RD advancement (RDO) process thoroughly. Beginning a couple of years back, most examines announced in [13]-[20] have concentrated on the learning approach for quick calculation because of the taking in property from the generally entangled measure of information to the best choice. Among these learning-based quick between coding calculations, CU size choice dependent on online SVM preparing [17] is probably the best approaches wherein three SVM classifiers utilized the primary gathering of pictures (GOP) encoded by utilizing $\mathrm{HM}$ as the preparation information. For include determination, misclassification-based element choice methodology is used to get three distinctive capabilities for profundity 0,1 and 2 . In any case, the preparation information of SVM classifiers are just from the principal GOP, making the classifier befuddle on the profundity choice and make a misclassification. Bogus positive (FP) is the misclassification when SVM classifier mistakenly chooses the parting $(+)$ choice for the present $\mathrm{CU}$ rather than the non-parting choice. Because of this, the RD costs for superfluous CUs are determined and Fuzzy SVM [17] can set aside a specific measure of effort for pointless profundity levels and may not successfully spare the computational weight of the quad tree-based $\mathrm{CU}$ parceling. Another significant circumstance is the hazard territory. In the event that an example is inside in the hazard zone, the first HM is set off that can expend the computational unpredictability of HEVC and may not altogether diminish the computational weight for chance region case. Another principle factor is the objective piece pace of rate control ( $\mathrm{RC}$ ) to allocate the necessary bits for input video arrangements. On the off chance that the objective piece rate is higher, the possibility of the parting choice can be higher. Thus, Fuzzy SVM may need to go to the profound profundity level and the quantity of CUs that should be determined the RD expenses might be higher. Along these lines, Fuzzy SVM may set aside a major computational effort for ascertaining the $\mathrm{RD}$ expenses of CUs at the high target bit rate. Moreover, no quick calculations referenced above think about discovering parceling example of a CTU as an improvement issue. All things considered, for finding an ideal CU parcel design from every single imaginable result can be displayed as a streamlining address and can be discovered an answer by an essentially incredible enhancer. A particle swarm optimizer (PSO) is a metaheuristic dependent on instruments of regular frameworks, for example, normal hereditary qualities and determination [23].By using the dual tree complex wavelet transform [25] one can reduce the time complexity of HEVC by reducing number of processing modes. To reduce complexity of HEVC Encoder, Hao Zhang, et. Al.,proposed a fast intra mode decision for the HEVC encoder. The overall fast intra mode decision algorithm consists of both micro- and macro-level schemes. At the micro-level, Author proposed the Hadamard cost based progressive rough mode search (pRMS) to selectively check the potential modes instead of traversing all candidates (i.e., up to 35 in HEVC). Fewer effective candidates will be chosen by the pRMS for the subsequent rate-distortion optimized quantization (RDOQ) to derive the 
rate-distortion (R-D) optimal mode. An early RDOQ skip method is also introduced to further the complexity reduction. At the macrolevel, author introduced the early coding unit (CU) split termination if the estimated R-D cost of (partial) sub-CUs is already larger than the R-D cost of the current CU. On average, the proposed fast intra mode decision provides about $2.5 \times$ speedup with just a $1.0 \%$ Bjontegaard delta rate (BD-rate) increase [28].

Venkatesh Munagala et. al [29] proposes a novel clustered entropy encoding scheme for high efficient computing systems for distributed video coding. As an exemplary system, high-efficiency video coding (HEVC) standard has been considered in this paper. This paper introduces clustered entropy computing, which distinguishes the video information has useful outliers. The pixel variations under varying frames are clustered based on the interestingness and the outliers are removed using an advanced entropy principle called as Holoentropy. The proposed encoding scheme is adopted in the HEVC and the simulations are carried out. The experimental results show that the quality of the video is preserved more than the conventional encoding for increased compression ratios.

In this paper, we propose a DTCWT based Intra Prediction along with improved entropy coding known as Holoentropy to decrease the computational complexity of HEVC and to further improves PSNR. These days, because of the higher casing pace of video groupings, for example, 50-60 edges for each second (fps) and up to $120 \mathrm{fps}$ for HD and UHD recordings, separately, the fleeting relationship between back-to-back edges is extraordinarily high. The fleeting connection alludes to the condition that the video information between progressive edges of a video arrangement are transiently related under a similar foundation scene with the equivalent moving articles. Because of these little interims, it is conceivable to share dividing examples of one casing to its back-to-back edges without seriously influencing the quality. Along these lines, outline level parcelling design sharing is one of our commitments to additionally brings down the computational weight of HEVC under a practically identical video quality. The next section describes our proposed approach for intraframe prediction. 1.Functional safety requirements

\section{Proposed Intra Frame Predition Algorithm}

The proposed method for the HEVC video coding by the intra coding is explained as follows. The block diagram of the proposed method is displayed in Fig 6.

As shown in the Figure 2, each frame in the video sequences is partitioned into coding tree units (CTUs). Then the partitioned blocks are given for the process of intra prediction.
CTU can be recursively divided into smaller coding units (CUs) using a quad-tree structure. A CU can be further partitioned to Prediction Units (PUs). In this approach, the encoder chooses the best intra prediction mode from the 35 angular modes that also include DC and Planar modes.

Initially, Dual Tree Discrete Wavelet Transform (DT-DWT) is applied to the partitioned video frames. By applying this transform, six oriented wavelet sub-bands are generated. Among the sub-bands, two LH and two HL sub-bands are used find the direction or angle of the texture in a block. Polarity of the texture angle is estimated with two HH Subbands.

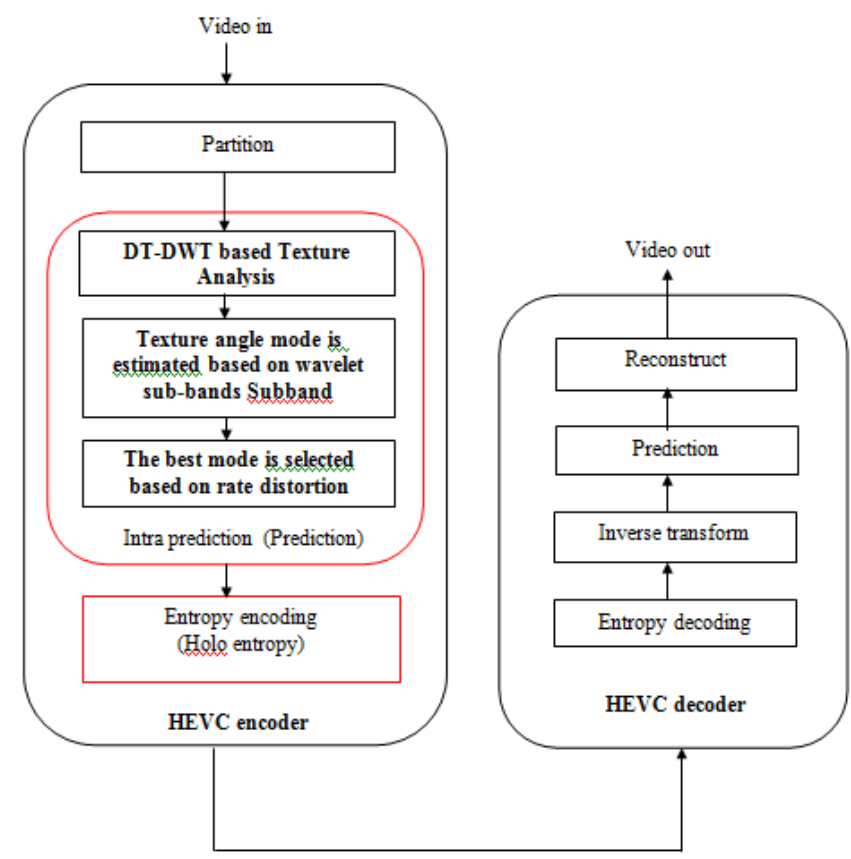

Fig.6: Proposed Intra Prediction Algorithm

With the direction and the angle of the texture, a mode is determined that is closer to the actual best mode. For best mode selection, the four modes around this determined mode $\left(\right.$ Mode $\left._{\text {deter }}\right)$, also DC and planar modes are considered as a final candidate list. This candidate list is forwarded to the process of Rate Distortion Optimization (RDO). With the RDO, the mode with minimum rate distortion cost is selected as the best mode (Mode $e_{\text {best }}$ ).

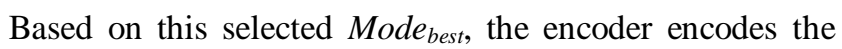
video frames. Besides, the performance of the entropy coding is improved by presenting the Holo entropy, which is used to remove the outlier pixels in each frame.

\section{A. Dual Tree-Discrete Wavelet Transform (DT-DWT)}

DT-DWT is the advanced design of DWT. Unlike DWT, DT-DWT can obtain better shift invariance and directional selectivity. DT-DWT is also known as complex transform 
since it includes the real and imaginary part of six oriented wavelet coefficients.

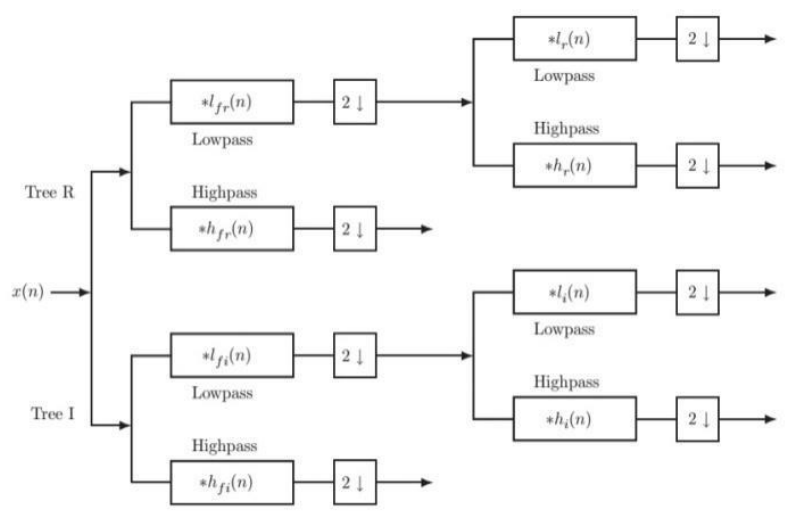

Fig7: Filter band Tree Structure of the DT-DWT

Fig. 7 shows the filter band tree structure of the DT-DWT. As shown in the figure, the top tree $\mathrm{R}$ generates the real parts of the DT-DWT coefficient and bottom tree I generates the imaginary parts of the DT-DWT coefficient. * denotes a convolution operation, $\downarrow 2$ means a down sampling by $2 . l_{f r}$ and $h_{f r}$ are low-pass filter and high-pass filter, which form a Hilbert transformed pair to insure the perfect reconstruction of the discrete wavelet transform. With the filter band tree structure, the following wavelet sub-bands which oriented at $\pm 75^{\circ}, \pm 15^{\circ}$ and \pm 450 are produced.

$$
\begin{aligned}
& \varphi 1(x, y)=\phi(x) \varphi(y) \quad \text { (LH Wavelet) } \\
& \varphi 2(x, y)=\varphi(x) \phi(y) \\
& \varphi 3(x, y)=\varphi(x) \varphi(y)
\end{aligned}
$$

From the above equations, $\phi(x)$ and $\phi(y)$ represent the low pass filter along with first and second dimension. Similarly, $\varphi(x)$ and $\varphi(y)$ represent the high pass filter along with first and second dimension. In addition, LH and HL sub-bands are oriented at vertical and horizontal directions respectively. The $\mathrm{HH}$ sub-band is simultaneously oriented along the $+45^{\circ}$ and $-45^{\circ}$ diagonal directions. It is also denoted as $\mathrm{HH}_{\mathrm{p}}$ (positive oriented direction) and $\mathrm{HH}_{\mathrm{n}}$ (negative oriented direction). Similarly, two LH sub-bands and two HL sub-bands are denoted as $\mathrm{LH}_{\mathrm{p}}, \mathrm{LH}_{\mathrm{n}}, \mathrm{HL}_{\mathrm{p}}$ and $\mathrm{HL}_{\mathrm{n}}$. With these six oriented sub-bands, best angular mode is estimated among the 35 angular modes for intra prediction process. The following section describes the selection of best mode.

\section{B. Angular mode selection}

Fig.8 shows the HEVC intra angular directions. As shown in the fig., HEVC intra prediction employs up to 35 modes for each intra-coding block, 33 angular modes, which predict the blocks, texture at specific directions, plus DC and planar modes for none

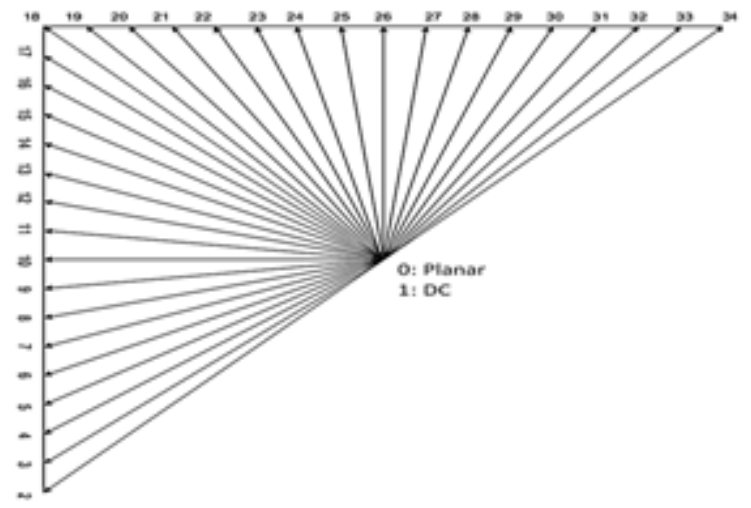

Fig. 8: HEVC intra angular directions

or less textured areas, based on the average of reference pixels. DC and planar are the two most probable modes in natural images which mainly contain plain areas, with no specific orientation. In standard HEVC, the encoder should decide between 35 intra prediction modes, and this decision should be made for each block at different sizes.

The main direction of the texture in each prediction block is calculated with the DT-DWT sub-bands. Direction (D) or angle of the texture is estimated by calculating the ratio between the total energy or variance of vertical and horizontal sub-bands. It can be calculated as eqation (1):

$D=\tan ^{-1}\left(\frac{v\left(L H_{p}\right)+v\left(L H_{n}\right)}{v\left(H L_{p}\right)+v\left(H L_{n}\right)}\right)$

Where, $V\left(L H_{p}\right)$ and $V\left(L H_{n}\right)$ represent the total variance of two LH sub-bands, $V\left(H L_{p}\right)$ and $V\left(H L_{n}\right)$ represent the total variance of two HL sub-bands. Polarity of this estimated texture direction or angle is defined with the two diagonal sub-bands $\mathrm{HH}_{\mathrm{p}}$ and $\mathrm{HH}_{\mathrm{n}}$ i.e., if $V\left(H H_{p}\right)$ is more than $V\left(H H_{n}\right)$, then the texture is more oriented towards the negative diagonal direction. Otherwise, the texture is more oriented towards the positive diagonal direction. For example, if the calculated texture angle is $53^{\circ}$ and is oriented towards the positive diagonal direction, then the mode 30 will be considered as the determined mode $\left(M_{\text {ode }}\right.$ deter $)$ shown in the Fig. 9. 


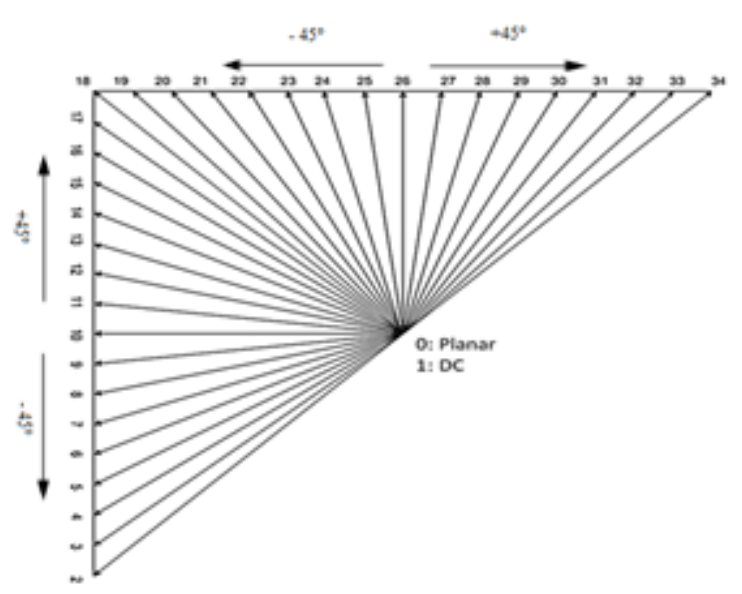

Fig. 9: HEVC Mode Determination

After finding the determined mode $\left(M o d e_{\text {deter }}\right)$, the best mode $\left(\right.$ Mode $\left._{\text {best }}\right)$ is selected around this mode. So the proposed encoder predicts one best mode around the determined mode instead of analyzing the all angular modes (33 angular modes). In this approach, six angular modes

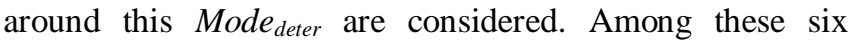
angular modes, two modes are added from the left of the Mode $_{\text {deter }}$ and other two modes are added from the right of the Mode $_{\text {deter }}$. Remaining most probable modes such as DC and planar modes are also included in this candidate list. This candidate list can be tabulated as shown in the TABLE II.

TABLE II Selected Candidate List for RDO process

\begin{tabular}{|c|c|c|c|c|c|c|}
\hline Mode $_{\text {deter }}$ & Mode $_{\text {deter }}$ & Mode $_{\text {deter }}$ & Mode $_{\text {deter }}$ & Mode $_{\text {deter }}$ & DC & Planar \\
$-(\mathrm{i}+1)$ & $-\mathrm{i}$ & & $+\mathrm{i}$ & $+(\mathrm{i}+1)$ & & \\
\hline
\end{tabular}

Finally, modes in the candidate list is taken into the account for the process of Rate Distortion Optimization (RDO). In this process, rate distortion cost is estimated for each mode in the candidate list. Rate distortion cost of a mode ( $\left.\mathrm{RD}_{\text {mode }}\right)$ is calculated as eqation (2):

$$
R D_{\text {mode }}=\left(S_{L u}+w_{C h} * S_{C h}+\lambda * T_{\text {mode }}\right)
$$

In above equation, $S$ defines the sum of square of transform coefficients of the differences between the pixels of the original block and reconstructed block. $T_{\text {mode }}$ represents the total bits in the mode for encoding process. $w_{C h}$ represents the weighting parameter for chroma-specific decisions and it can be calculate based on the Quantization Parameters (QP) as equation(3):

$$
w_{\text {chroma }}=2^{(\mathrm{QP}-\mathrm{QP} \text { chroma }) / 3}
$$

Also, $\lambda$ represents the Lagrange multiplier and it can be calculated as equation (4):

$$
\lambda=0.85 \times 2^{(Q P-12) / 3}
$$

Finally, the mode with minimum rate distortion cost is considered as the best mode $\left(\right.$ Mode $_{\text {best }}$ ) of texture angle or direction.

\section{C. CABAC Coding in HEVC}

In video codec implementation, $\mathrm{CABAC}$ seems to be a throughput bottleneck [29]. Depending on the number of bins that it processes per second, the throughput of CABAC can be estimated. Increase in a number of bins is directly proportional to increase in throughput. However, it is very hard to handle multiple bins parallel in CABAC. For recursive interval division, the updated range is fed back and for the correct probability estimate, the updated context is fed back. Depending on the binIdx and type of the syntax element, the selection of context is done. Both the feedback loops-context update and range update is very easily understood than the context selection loops. The computation efforts are needed, if the bin context depends on the other bin value. This may lead to area cost increment and delay in the critical path.

Overcoming the throughput problem of CABAC is important. Various techniques such as Holoentropy are developed for improving throughput with minimum loss of coding. By reducing the context coded bins, it is easier to process the coded bins in parallel and so the throughput can be improved.

\section{D. Entropy Coding based on Holoentropy}

Definition 1 [29] (Holoentropy of a random vector) The holoentropy $\operatorname{HLx}(y)$ is defined as the sum of the entropy and the total correlation of the random vector $\mathrm{Y}$, and can be expressed by the sum of the entropies on all attributes.

$$
H L_{x}(Y)=H_{x}(Y)+C_{x}(Y)=\sum_{i=1}^{m} H_{x}\left(y_{i}\right)
$$

where, $\operatorname{HLx}(\mathrm{Y})$ is the Holoentropy model, $\mathrm{Y}$ is the mutual information of multivariate discrete random vectors, $\mathrm{m}$ is the number of attributes, $\mathrm{Hx}(\mathrm{Y})$ is the Entropy of $\mathrm{Y}, \mathrm{Cx}(\mathrm{Y})$ is the total correlation and yiis the categorical attribute.

\section{Proposition 1:}

$0 \leq H L_{x}(Y) \leq m \log (n)$. 
Proof: For yi of $Y$, the minimum entropy of the attribute satisfies $H x(y i)=0$, if all the values seems to be same and if the values are different, the maximum entropy is given as $H x(y i)=\log (n)$. The inequalities hold because $H L x(Y)<=$ $m \log (n)$.

Definition 2 [29] Weighted Holoentropy of a random vector. The weighted holoentropy $\mathrm{Wx}(\mathrm{Y})$ is the sum of the weighted entropy on each attribute of the random vector $Y$.

$$
W_{x}(Y)=\sum_{i=1}^{m} w_{x}\left(y_{i}\right) H_{x}\left(y_{i}\right) \text {. }
$$

\section{E. HEVC Decoding}

Inverse operation is carried out in the decoder side. Firstly, Entropy decoding is carried out followed by inverse transform and de quantization. Reconstruction of residue is carried out and finally original video is obtained at the decoder output.

\section{Result Evaluation}

To evaluate the performance of the proposed Intra Prediction Algorithm, the algorithm is implemented in Net Beans IDE 8.2. As explained in the above chapter, proposed algorithm uses two level discrete wavelet transform for finding out the best-estimated mode. Then Candidate list of mode is prepared including best-estimated mode, nearby modes, DC and Planer mode. Rate distortion Optimization is applied on candidate list and best mode is selected. The performance of the entropy coding is improved by presenting the Holo entropy, which is used to remove the outlier pixels in each frame.

The evaluation metrics employed are PSNR, Encoding time (ET), CCPS (Clock Cycles per Second) and MSE. Frame rate is set at $30 \mathrm{fps}$.

\section{A. Dataset description}

In this proposed methodology, we used Jockey video. The resolution of of Jockey video is $4096 \times 2160$ pixels and the frame rate is $120 \mathrm{fps}$. The length of Jockey video is $5 \mathrm{~s}$.

\section{B. Encoder Complexity Reduction}

TABLE III results and comparison of proposed intra prediction algorithm with Reference [31] in terms of PSNR, CCPS, ET, MSE for Jockey video. With the proposed Intra Prediction algorithm, encoder complexity in terms of encoding time for the test sequence is reduced as compared to reference [31].
TABLE III. Results and Comparison of Proposed Intra Prediction Algorithm with Reference [31] in terms of PSNR, CCPS, ET, MSE for Jockey video

\begin{tabular}{|c|c|c|c|c|c|}
\hline $\begin{array}{l}\text { Testing } \\
\text { Parameters } \\
\text { Algorithn } \\
\text { Applied }\end{array}$ & MSE & PSNR & CCPS & ET & $\begin{array}{l}\text { Total } \\
\text { Time }\end{array}$ \\
\hline $\begin{array}{l}\text { Two stage } \\
\text { fast mode } \\
\text { decision } \\
\text { algorithm } \\
\text { for intra } \\
\text { prediction } \\
\text { in } \\
\text { HEVC[31] }\end{array}$ & 2.10603 & 39.06 & 154452011 & 454656 & $\begin{array}{l}7 \\
\text { minutes } \\
45 \\
\text { seconds }\end{array}$ \\
\hline $\begin{array}{l}\text { Proposed } \\
\text { Mode } \\
\text { Selective } \\
\text { based Intra } \\
\text { Prediction } \\
\text { Algorithm }\end{array}$ & 1.50141 & 46.39 & 154444293 & 360256 & $\begin{array}{l}4 \\
\text { minutes } \\
4 \\
\text { seconds }\end{array}$ \\
\hline
\end{tabular}

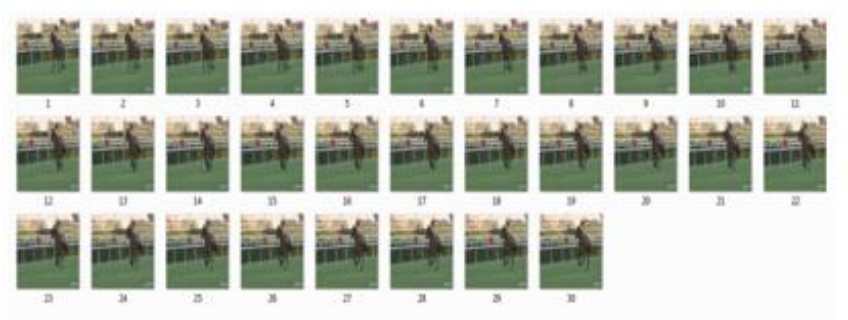

Fig.10. Input Frames used in Execution of both Reference [31] and Proposed Algorithm

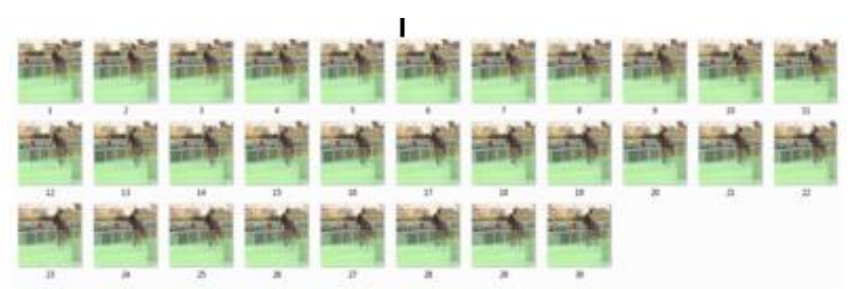

Fig.11. Output Frames obtained after Executing Reference Paper Algorithm [31]

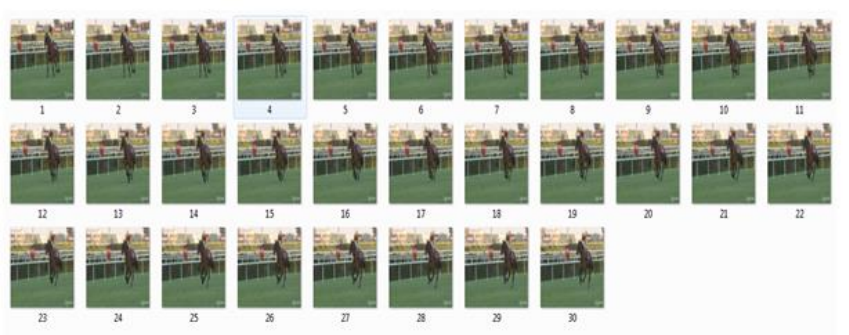

Fig.12. Output Frames obtained after Executing Proposed Algorithm:

From the above result, it is clear that by using two-stage reference algorithm along with two level discrete wavelet transform, coding efficiency can be increased with decreased computational complexity. 
The conclusion and some interesting observations from these results are mentioned in the next section.

\section{Conclusion and Future Work}

Encoding complexity in HEVC increases greatly because the rate-distortion optimization process, which is, be performed for all combinations of CU, PU, and TU to obtain the optimal CTU partition. This increased complexity is a major problem especially for power constrained devices or real-time applications especially for high-resolution videos. Therefore, it is highly desirable to optimize the encoding process for computational complexity reduction while maintaining the coding efficiency of HEVC.

In this paper, a method called selective Mode based intra prediction algorithm is proposed. Proposed algorithm uses two level discrete wavelet transform for finding out the bestestimated mode. Then Candidate list of mode is prepared including best-estimated mode, nearby modes, DC and Planer mode. Rate distortion Optimization is applied on candidate list and best mode is selected. Finally, quantization and entropy coding is performed to compress the input video signal. The performance of the entropy coding is improved by presenting the Holo entropy, which is used to remove the outlier pixels in each frame. The experimental results are conducted for Jockey video test sequences for 30 frames. The results are evaluated based on encoding time and peak signal to noise ratio (PSNR). The results of comparative experiments demonstrate that the proposed algorithm can effectively reduce the computational complexity of HEVC Encoder.

There are many other ways to explore in the CU early termination and fast intra prediction in the intra prediction area as suggested by literature. In future, many of these methods can be combined, or if needed, one method may be replaced by a new method and encoding time gains can be explored. Similar Intra Prediction algorithms can be developed for fast inter-prediction resulting in less encoding time and reduced complexity.

This work can be further improved by evaluating the performance for higher bit rate videos. Moreover, in order to really optimize the performance further, researchers can use quantum computing for processing, and develop quantum computational layers in order to evaluate its performance, and apply the proposed machine.

\section{References:}

[1]. G. Sullivan et al., "Overview of the high efficiency video coding (HEVC) standard", IEEE Transactions on Circuits and Systems for Video Technology, Vol. 22, No. 12, pp 1649-1668, December 2012.
[2]. T. Wiegand, G. J. Sullivan, G. Bjontegaard, and A. Luthra, "Overview of the H.264/AVC video coding standard," IEEE Transactions on Circuits and Systems for Video Technology, vol. 13, no. 7, pp. 560-576, Jul. 2003.

[3]. JCT-VC, HM Software. Accessed: Nov. 5, 2016. [Online].

https://hevc.hhi.fraunhofer.de/svn/svn_HEVCSoftware/tags/ HM-16.5/

[4]. G. J. Sullivan and J.-R. Ohm, "Recent developments in standardization of high efficiency video coding (HEVC)," SPIE Trans. on Applications of Digital Image Processing XXXIII, Proceedings of SPIE, A. G. Tescher, Ed., vol. 7798,no. 7798-30, August 2010.

[5]. Harshdeep Brahmasury Jain, "Fast Intra Mode Decision in High Efficiency Video Coding," Ph. D. Thesis, The University of Texas at Arlington, August 2014.

[6]. Vinoothna Gajula, "Complexity Reduction in HEVC Intra Coding and Comparison with H.264/AVC," Master of Science Thesis in Electrical Engineering, The University of Texas at Arlington, December 2013.

[7]. T. Zhang, M.-T. Sun, D. Zhao, and W. Gao, "Fast intramode and CU size decision for HEVC,', IEEE Transactions on Circuits and Systems for Video Technology, vol. 27, no. 8, pp. 1714-1726, Aug. 2017.

[8]. L. Shen, Z. Liu, X. Zhang, W. Zhao, and Z. Zhang, "An effective CU size decision method for HEVC encoders," IEEE Trans. on Multimedia, vol. 15, no. 2, pp. 465-470, Feb. 2013.

[9]. X. Hou and Y. Xue, "Fast coding unit partitioning algorithm for HEVC,' in Proc. IEEE Int. Conf. Consum. Electron. (ICCE), Jan. 2014, pp. 7-10. .

[10]. I. Zupancic, S. G. Blasi, E. Peixoto, and E. Izquierdo, "Inter-prediction optimizations for video coding using adaptive coding unit visiting order,' IEEE Trans.on Multimedia, vol. 18, no. 9, pp. 1677-1690, Sep. 2016.

[11]. X. Shen, L. Yu, and J. Chen, "Fast coding unit size selection for HEVC based on Bayesian decision rule,' in Proc. of Picture Coding Symp., May 2012, pp. 453-456. 
[12]. K. Duan, P. Liu, K. Jia, and Z. Feng, “An adaptive quad-tree depth range prediction mechanism for HEVC," IEEE Access, vol. 6, pp. 54195-54206, 2018.

[13]. X. Shen and L. Yu, "CU splitting early termination based on weighted SVM,' EURASIP Journal proceedings of Image and Video Processing, vol. 2013, no. 1, p. 4, Dec. 2013.

[14]. G. Corrêa, P. A. Assuncao, L. V. Agostini, and L. A. da Silva Cruz, "Fast HEVC encoding decisions using data mining," IEEE Transactions on Circuits and Systems for Video Technology, vol. 25, no. 4, pp. 660-673, Apr. 2015.

[15]. Y. Zhang, S. Kwong, X. Wang, H. Yuan, Z. Pan, and L. $\mathrm{Xu}$, "Machine learning-based coding unit depth decisions for flexible complexity allocation in high efficiency video coding," IEEE Transaction on Image Processing, vol. 24, no. 7, pp. 2225-2238, Jul. 2015.

[16]. A. Heindel, T. Haubner, and A. Kaup, "Fast CU split decisions for HEVC inter coding using support vector machines," in Proc. Picture Coding Symp. (PCS), Dec. 2016, pp. 1-5.

[17]. L. Zhu, Y. Zhang, S. Kwong, X. Wang, and T. Zhao, "Fuzzy SVM-based coding unit decision in HEVC,', IEEE Transaction on Broadcasting, vol. 64, no. 3, pp. 681-694, Sep. 2018.

[18]. H.-S. Kim and R.-H. Park, "Fast CU partitioning algorithm for HEVC using an online-learning-based Bayesian decision rule," IEEE Transactions on Circuits and Systems for Video Technology, vol. 26, no. 1, pp. 130138, Jan. 2016.

[19]. L. Zhu, Y. Zhang, Z. Pan, R. Wang, S. Kwong, and Z. Peng, "Binary and multi-class learning based low complexity optimization for HEVC encoding," IEEE Transaction on Broadcasting, vol. 63, no. 3, pp. 547-561, Sep. 2017.

[20]. M. Xu, T. Li, Z. Wang, X. Deng, R. Yang, and Z. Guan, "Reducing complexity of HEVC: A deep learning approach,' IEEE Transaction on Image Processing, vol. 27, no. 10, pp. 5044-5059, Oct. 2018.

[21]. Z. Pan, S. Kwong, M.-T. Sun, and J. Lei, "Early MERGE mode decision based on motion estimation and hierarchical depth correlation for HEVC,' IEEE Transaction on Broadcasting, vol. 60, no. 2, pp. 405-412, Jun. 2014.
[22]. Z. Pan, J. Lei, Y. Zhang, X. Sun, and S. Kwong, "Fast motion estimation based on content property for lowcomplexity H.265/HEVC encoder,' IEEE Transaction on Broadcasting, vol. 62, no. 3, pp. 675-684, Sep. 2016.

[23]. M. Mitchell, An Introduction to Genetic Algorithms. Cambridge, MA, USA: MIT Press, 1996.

[24]. Z. Michalewicz, Genetic Algorithms+Data Structures=Evolution Programs, 3rd ed. Berlin, Germany: Springer-Verlag, 1996.

[25]. Farhad Pakdaman, Mahmoud-Reza Hashemi, and Mohammad Ghanbari, "Fast and Efficient Intra Mode Decision for HEVC, Based on Dual-Tree Complex Wavelet," in Proc. Of Article in Multimedia Tools and Applications, May 2016

[26]. Romberg J-K, Hyeokho C, Baraniuk R-G (2001) Multiscale edge grammars for complex wavelet transforms. Proc. International Conference on Image Processing (ICIP), pp. 614-617

[27]. Selesnick I-W, Baraniuk R-G, Kingsbury N-C (2005) The dual-tree complex wavelet transform. IEEE Signal Process Mag 22(6):123-151

[28]. Hao Zhang, Zhan Ma, "Fast Intra Mode Decision for High Efficiency Video Coding (HEVC)," IEEE Trans. on Circuits and Systems for Video Technology, vol. 24, no. 4, April 2014, pp.660-668.

[29]. Venkatesh Munagala, K. Satya Prasad, "Clustered entropy computing: a holoentropy based encoding scheme for high efficient computing systems," transactions on Multimedia Tools and Applications, Springer Science+ Business Media, March 2018

[30]. S. Vasudevan and K. R. Rao "Combination method of fast HEVC encoding" in Proc. of IEEE International Conference of Electrical Engineering/Electronics, Computer, Telecommunication and Information Technology (ECTI-CON), IEEE, May 2014, Korat, Thailand, pp. 1-6.

[31]. Mohammadreza Ramezanpour Fini \& Farzad Zargari , "Two stage fast mode decision algorithm for intra prediction in HEVC," transactions on Multimedia Tools and Applications, Springer Science+ Business Media, May 2015, New York 
[32]. https://www.intechopen.com/books/recent-advancesin-image-and-video-coding/efficient-coding-tree-unit-ctudecision-method-for-scalable-high-efficiency-video-codingshvc-encode

[33]. https://www.researchgate.net/figure/Diagram-ofHEVC-standard-decoder fig1 320826067 